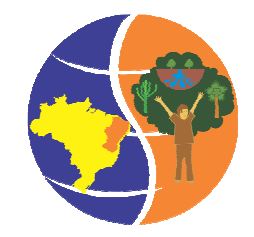

\author{
III Simpósio Brasileiro de Recursos Naturais do Semiárido - SBRNS \\ "Manejo de bacias hidrográficas em regiões semiáridas: potencialização da produção de água" \\ Fortaleza - Ceará, Brasil \\ 20 a 22 de junho de 2017
}

\title{
NECESSIDADE HÍDRICA DO ABACAXI ORNAMENTAL EM CULTIVO PROTEGIDO
}

\section{Janderson Pedro da Silva ${ }^{1}$, Arthur Abreu Alves Marques ${ }^{2}$, Rubens Sonsol Gondim Fernando José Hawerroth ${ }^{4}$, Ana Cristina Portugal Pinto de Carvalho ${ }^{5}$}

${ }^{1}$ Estágio em Agronomia, Embrapa, (85) 3391-7310, jandersonpedro1@ gmail.com

${ }^{2}$ Estágio em Geografia, Embrapa, (85) 3391-7206, abreuarthur@yahoo.com.br

${ }^{3}$ Doutor, Embrapa Agroindústria Tropical, 3391-7206, rubens.gondim@embrapa.br

${ }^{4}$ Doutor, Embrapa Uva e Vinho, 3391-7206, fernando.hawerroth@embrapa.br

5 Doutora, Embrapa Agroindústria Tropical, 3391-7272, cristina.carvalho@embrapa.br

RESUMO: O abacaxi ornamental irrigado é uma espécie que vem apresentando importância econômica como flor de corte e recentemente para comercialização em vaso, sem haver, entretanto, informações sobre as necessidades hídricas das plantas. O presente trabalho teve como objetivo estimar as necessidades hídricas do híbrido Ananás comosus var. bracteatus x A. comosus var. erectifolius, cultivado em vaso com substrato, em ambiente protegido, tipo telado. $\mathrm{O}$ experimento foi realizado na área experimental da Embrapa Agroindústria Tropical, no município de Pacajus, CE, envolvendo 1.035 vasos de $0,14 \mathrm{~m}$ de diâmetro, dispostos sobre mesas de ferro galvanizado de $5,68 \mathrm{~m}^{2}$, distanciados de $0,07 \mathrm{~m}$ e totalizando aproximadamente 20 vasos por metro quadradoAplicou-se o método do balanço hídrico, utilizando coletores instalados sob os vasos. A cultura foi irrigada a cada dois dias, manualmente em volume determinado de $300 \mathrm{~mL}$. A evapotranspiração acumulada durante a condução do experimento foi de $291,0 \mathrm{~mm}$, correspondendo a um valor médio de $0,53 \mathrm{~mm} \mathrm{dia}^{-1}$. Observaram-se variações nos valores de coeficiente de cultivo $(\mathrm{Kc})$ decorrentes das condições climáticas e dos estádios fenológicos da cultura. Os valores médios dos coeficientes de cultivo (Kcs), obtidos pela evapotranspiração de referência estimada pela equação de Penman-Monteith e variáveis climáticas medidas (temperaturas máxima e mínima, radiação solar, velocidade do vento, umidade relativa e precipitação pluvial) por uma estação meteorológica instalada no interior do telado, são 0,$13 ; 0,13$ a 0,51 e 0,51 para os estádios inicial (0-45 dias após o plantio - DPA); intermediário (46 a 66 DAP) e vegetativo (67 a 211 DAP), respectivamente.

PALAVRAS-CHAVE: Ananás comosus var. bracteatus x A. comosus var. erectifolius, evapotranspiração, coeficientes de cultivo $(\mathrm{Kc})$, balanço hídrico.

\section{GREENHOUSE ORNAMENTAL PINEAPPLE CROP WATER NEEDS}

ABSTRACT: Irrigated ornamental pineapple is a vegetable specie which economic importance is becoming greater as cut flower or sold in vase, with no information about crop water needs. The objetctive of this task was to estimate Ananás comosus var. bracteatus x A. comosus var. erectifolius cultivated in greenhouse vases filled with substrate water needs. The experiment was conducted in Embrapa Agroindústria Tropical station in Pacajus, CE, involving 1.035 vases of 0,14 $\mathrm{m}$ diameter distributed over iron tables of $5,68 \mathrm{~m}^{2}$, distanced of $0,07 \mathrm{~m}$ and totalizing about 20 vases per squared meter. Water balance method was applied using water collectors under the vases. The crop was irrigated each 2 days by applying $300 \mathrm{~mL}$ per plant. The evapotranspiration 
accumulated during the experiment was $291,0 \mathrm{~mm}$, corresponding $0,53 \mathrm{~mm} \mathrm{dia}^{-1}$. Variation on crop coefficient $(\mathrm{Kc})$ was observed due to climate conditions and crop development stage. Average crop coefficients (Kcs) estimated by FAO-Penman-Monteith reference evapotranspiration and climate variables (maximum and minimum temperature, solar radiation, wind speed, relative humidity and rainfall) measured by meteorological station installed in the greenhouse are 0,$13 ; 0,13$ to 0,51 and 0,51 for initial (0-45 days after planting - DPA); intermediate (46 to 66 DAP) and development stage (67 to 211 DAP), respectively.

KEYWORDS: Ananás comosus var. bracteatus x A. comosus var. erectifolius, evapotranspiração, crop coefficient $(\mathrm{Kc})$, water balance

\section{INTRODUÇÃO}

O abacaxi ornamental irrigado é uma espécie que vem apresentando importância econômica como flor de corte e recentemente para comercialização em vaso, sem haver, entretanto, informações sobre as necessidades hídricas das plantas. Como a irrigação é um trato cultural indispensável no semiárido e que eleva a produtividade, se faz necessário à quantificação da necessidade hídrica da cultura frente ao efeito dos fatores climáticos. Assim, estimar a evapotranspiração e coeficientes de cultivo são necessários para um eficiente controle de irrigação, evitando desperdícios de água e nutrientes.

O Abacaxi Ornamental (Ananás comosus var. bracteatus) é uma planta originária das regiões tropicais e subtropicais da América do sul, pertencente à família das Bromeliaceaes. São plantas ornamentais que se adaptam bem à condição de cultivo em vasos, e se destacam pela beleza e aceitação de mercado. Seus frutos, embora não utilizados na alimentação, também são vistosos e possuem potencial ornamental.

Segundo Carvalho (2014), o abacaxi ornamental é uma planta herbácea, perene, rizomatosa, com altura variando entre $50 \mathrm{~cm}$ e $120 \mathrm{~cm}$, com folhas largas, compridas e com espinhos afilados, além de flores com brácteas longas cobrindo o ovário, grosseiramente serrilhadas, geralmente vermelhas ou rosas.

Pode ser cultivado nas regiões entre o paralelo $25^{\circ} \mathrm{N}$ e $25^{\circ} \mathrm{S}$, a temperatura de cultivo considerada ideal está entre $22^{\circ}$ e $32^{\circ}$, e seu requerimento de luminosidade ótimo está entre 2500 e 3000 horas por ano ( Matos et al., 2006).

O presente trabalho objetivou fornecer aos técnicos e produtores informações sobre as necessidades hídricas aplicáveis ao manejo de irrigação na cultura do Ananás comosus var. bracteatus. 


\section{MATERIAL E MÉTODOS}

Os coeficientes de cultivo do abacaxi ornamental foram determinados em experimento realizado em Campo Experimental, pertencente a Embrapa Agroindústria Tropical, localizado no município de Pacajus, CE. O município está localizado a $4^{\circ} 10^{\prime} 26^{\prime \prime}$ de latitude Sul e a $38^{\circ}$ 27' 50" longitude Oeste. O clima da região, segundo a classificação Köppen é Aw', classificado como tropical chuvoso, clima de savana e caracterizado por apresentar o máximo de chuvas no outono e período seco no inverno.

O experimento foi conduzido no interior de um ambiente protegido com taxa de sombreamento de $50 \%$ envolveu 1.035 vasos de $0,14 \mathrm{~m}$ de diâmetro dispostos sobre mesas de ferro galvanizado de $5,68 \mathrm{~m}^{2}$, distanciados de $0,07 \mathrm{~m}$ e totalizando aproximadamente 20 vasos por $\mathrm{m}^{2}$.

O substrato utilizado no experimento foi uma mistura comercial composta de casca de pinus composta, vermiculita expandida, palha de arroz, corretivo de acidez e superfosfato simples em pó acrescido de NPK e micronutrientes.

Aplicou-se o método do balanço hídrico, utilizando coletores instalados sob os vasos. A cultura foi irrigada a cada dois dias, com volume determinado de $300 \mathrm{~mL}$. O manejo da irrigação foi feito por meio do consumo de água ou evapotranspiração da cultura (ETc).

$\mathrm{O}$ coeficiente de cultivo $(\mathrm{Kc})$, que relaciona a evapotranspiração de referência à evapotranspiração máxima da cultura, que ocorre em condições ótimas de suprimento hídrico foi estimado pela equação:

$\mathrm{ETc}=\mathrm{ETo} \times \mathrm{Kc}$

em que: ETc - Evapotranspiração da cultura $\left(\mathrm{mm} \mathrm{dia}^{-1}\right)$; ETo - Evapotranspiração de referência $\left(\mathrm{mm} \mathrm{dia}^{-1}\right)$, e Kc - Coeficiente de Cultivo.

Para a determinação da Evapotranspiração da cultura, foi utilizado o método de balanço hídrico, representado pela equação a seguir:

$\mathrm{ETc}=\mathrm{P}+\mathrm{I} \pm \mathrm{Qz}-\Delta \mathrm{A}$ 
em que: ETc - evapotranspiração da cultura (mm); P - precipitação pluvial (mm); I - irrigação (mm); Qz - drenagem profunda ou ascensão capilar (mm), e $\Delta \mathrm{A}$ - variação do armazenamento da água do solo.

Para estimativa da Evapotranspiração de referência foi utilizada a equação FAO/Penman-Monteith, empregando dados diários de temperatura máxima e mínima do ar, umidade relativa do ar, radiação e velocidade do vento medida a dois metros de altura, conforme equação abaixo:

$$
\mathrm{ET}_{\mathrm{o}^{\mathrm{PM}}}=\frac{0,408 \Delta(\mathrm{Rn}-\mathrm{G})+\gamma \frac{900}{\mathrm{~T}+273} \mathrm{u}_{2}\left(\mathrm{e}_{\mathrm{s}}-\mathrm{e}_{\mathrm{a}}\right)}{\Delta+\gamma\left(1+0,34 \mathrm{u}_{2}\right)}
$$

em que: ETo - evapotranspiração de referencia, $\mathrm{mm} \mathrm{dia}^{-1} ; \mathrm{Rn}$ - radiação liquida na superfície das culturas, MJ m2 dia $^{-1}$; G - fluxo de calor no solo, MJ m2 dia ${ }^{-1}$; $\mathrm{T}$ - média diária da temperatura do ar a $2 \mathrm{~m}$ de altura, ${ }^{\circ} \mathrm{C}$; $\mathrm{u} 2$ - velocidade do vento a $2 \mathrm{~m}$ de altura, $\mathrm{m} \mathrm{s}^{-1}$; es pressão da saturação de vapor, $\mathrm{kPa}$; ea - pressão de vapor atual, $\mathrm{kPa}$; es - ea - déficit de saturação de vapor, $\mathrm{kPa}$; D - inclinação da curva da pressão de vapor versus temperatura, $\mathrm{kPa}^{\circ} \mathrm{C}^{-1}$, e $\gamma$ - constante psicrométrica, $\mathrm{kPa} \mathrm{oC}^{-1}$.

Os dados climáticos utilizados no trabalho foram coletados na estação meteorológica instalada no interior da casa de vegetação, conforme Tabela 1 .

Tabela 1. Médias mensais de umidade relativa, temperatura máxima, mínima e média, precipitação, velocidade do vento e radiação (Pacajus, CE, 2014 - 2015).

\begin{tabular}{ccccccccc}
\hline Mês/Ano & $\begin{array}{c}\text { Umidade } \\
\text { Relativa } \\
(\%)\end{array}$ & $\begin{array}{c}\text { Tmáx } \\
\text { Média } \\
\left({ }^{\circ} \mathrm{C}\right)\end{array}$ & $\begin{array}{c}\text { Tmín } \\
\text { Média } \\
\left({ }^{\circ} \mathrm{C}\right)\end{array}$ & $\begin{array}{c}\text { Temperatura } \\
\text { Média } \\
\left({ }^{\circ} \mathrm{C}\right)\end{array}$ & $\begin{array}{c}\text { Precipitação } \\
(\mathrm{mm})\end{array}$ & $\begin{array}{c}\text { Irrigação } \\
(\mathrm{mm})\end{array}$ & $\begin{array}{c}\text { Velocidade } \\
\text { do Vento } \\
\left(\mathrm{m}_{\mathrm{s} \mathrm{dia}}{ }^{-1}\right)\end{array}$ & $\begin{array}{c}\text { Radiação } \\
\left(\mathrm{MJ}^{-1} \mathrm{~m}^{2}\right. \\
\left.\text { dia }^{-1}\right)\end{array}$ \\
\hline JUN/14 & 79 & 35 & 22,7 & 27,4 & 0 & 2 & 26,9 & 5,6 \\
JUL/14 & 75,8 & 34,9 & 22,1 & 27,3 & 1,2 & 2 & 34,5 & 7,9 \\
AGO/14 & 70 & 35,7 & 21,7 & 27,7 & 0,3 & 2 & 45,8 & 6,2 \\
SET/14 & 71 & 35,5 & 23 & 28,1 & 0,3 & 2 & 51,7 & 4,4 \\
OUT/14 & 69 & 35,9 & 23,3 & 28,7 & 0,8 & 2 & 53,4 & 5,2 \\
NOV/14 & 74,1 & 35 & 22,7 & 28,1 & 2,8 & 2 & 40,3 & 4,5 \\
DEZ/14 & 73,6 & 35,2 & 23,3 & 28,4 & 0,5 & 2 & 41,3 & 4 \\
JAN/15 & 74,9 & 33,9 & 23,2 & 27,8 & 3,6 & 2 & 32,6 & 3,5 \\
\hline Média & 73,4 & 35,1 & 22,8 & 27,9 & 1,2 & 2 & 40,8 & 5,2 \\
\hline
\end{tabular}




\section{RESULTADOS E DISCUSSÃO}

Na Tabela 2 estão apresentados os valores médios semanais de ETo, ETc e Kc do abacaxi ornamental. As variações dos valores de Kc ocorreram devido à variação das condições climáticas predominantes e estádios fenológicos da cultura. A evapotranspiração acumulada durante a condução do experimento foi da ordem de $22,82 \mathrm{~mm}$, correspondendo a um valor médio de $0,76 \mathrm{~mm} \mathrm{semana}^{-1}$.

Tabela 2. Valores de evapotranspiração da cultura (ETc), evapotranspiração de referencia (ETo) e coeficiente de cultivo (Kc) do abacaxi ornamental cultivado com $50 \%$ de sombreamento, na região de Pacajus, CE.

\begin{tabular}{|c|c|c|c|c|}
\hline DAP & Estádios Fenológicos & $\begin{array}{c}\text { ETc } \\
\left(\mathrm{mm} \mathrm{semana}^{-1}\right)\end{array}$ & $\begin{array}{c}\text { ETo } \\
\left(\mathrm{mm} \mathrm{semana}^{-1}\right)\end{array}$ & $(\mathrm{Kc})$ \\
\hline 7 & Inicial & 0,27 & 7,20 & 0,04 \\
\hline 14 & Inicial & 0,25 & 7,22 & 0,03 \\
\hline 21 & Inicial & 3,99 & 5,02 & 0,10 \\
\hline 28 & Inicial & 0,24 & 0,00 & 0,10 \\
\hline 35 & Inicial & 0,25 & 2,06 & 0,12 \\
\hline 42 & Inicial & 0,23 & 7,28 & 0,03 \\
\hline 49 & Intermediário & 0,24 & 7,28 & 0,03 \\
\hline 56 & Intermediário & 0,30 & 6,59 & 0,04 \\
\hline 63 & Intermediário & 0,54 & 1,73 & 0,31 \\
\hline 70 & Vegetativo & 0,25 & 1,45 & 0,17 \\
\hline 77 & Vegetativo & 1,15 & 1,74 & 0,66 \\
\hline 84 & Vegetativo & 3,53 & 1,65 & 0,31 \\
\hline 91 & Vegetativo & 1,14 & 1,76 & 0,65 \\
\hline 98 & Vegetativo & 0,25 & 1,71 & 0,45 \\
\hline 105 & Vegetativo & 0,25 & 1,80 & 0,14 \\
\hline 112 & Vegetativo & 0,30 & 2,08 & 0,14 \\
\hline 119 & Vegetativo & 0,53 & 1,91 & 0,28 \\
\hline 126 & Vegetativo & 0,21 & 1,92 & 0,11 \\
\hline 133 & Vegetativo & 0,23 & 1,59 & 0,84 \\
\hline 140 & Vegetativo & 0,23 & 1,54 & 0,84 \\
\hline 147 & Vegetativo & 1,44 & 1,50 & 0,97 \\
\hline 154 & Vegetativo & 0,84 & 1,57 & 0,53 \\
\hline 161 & Vegetativo & 0,85 & 1,45 & 0,59 \\
\hline 168 & Vegetativo & 2,04 & 1,59 & 1,28 \\
\hline 175 & Vegetativo & 0,24 & 1,58 & 0,15 \\
\hline 182 & Vegetativo & 0,21 & 1,51 & 0,14 \\
\hline 189 & Vegetativo & 0,85 & 1,25 & 0,68 \\
\hline 196 & Vegetativo & 0,16 & 1,07 & 0,56 \\
\hline 203 & Vegetativo & 0,30 & 1,66 & 0,56 \\
\hline 210 & Vegetativo & 1,50 & 0,42 & 0,56 \\
\hline
\end{tabular}


Na Figura 1 está representada a curva do coeficiente de cultivo $(\mathrm{Kc})$ determinada para a cultura, o qual apresentou um valor médio de 0,07 na fase de crescimento inicial. Durante a fase de crescimento intermediário o Kc apresentou um valor de 0,13. No estádio vegetativo ocorreu um rápido aumento apresentando um Kc médio de 0,51.

Em experimento conduzido nos Tabuleiros Costeiro da Paraíba, Azevedo et al. (2007) identificou valores de Kc para fase vegetativa do abacaxi comestível (Ananas comosus L. var. Pérola) superiores (140 a 308 DAP) I $(0,88)$ e II $(0,91)$. Já Santana et al. (2013), para as condições de Uberaba-MG, encontraram valores de 0,57 e 0,66 para as fases inicial e vegetativa, respectivamente, aproximando-se do determinado neste trabalho $(0,51)$. As diferenças dos resultados para o abacaxi ornamental podem ser atribuídas por tratar-se de uma outra espécie, de menor porte e mais adaptada ao clima semiárido.

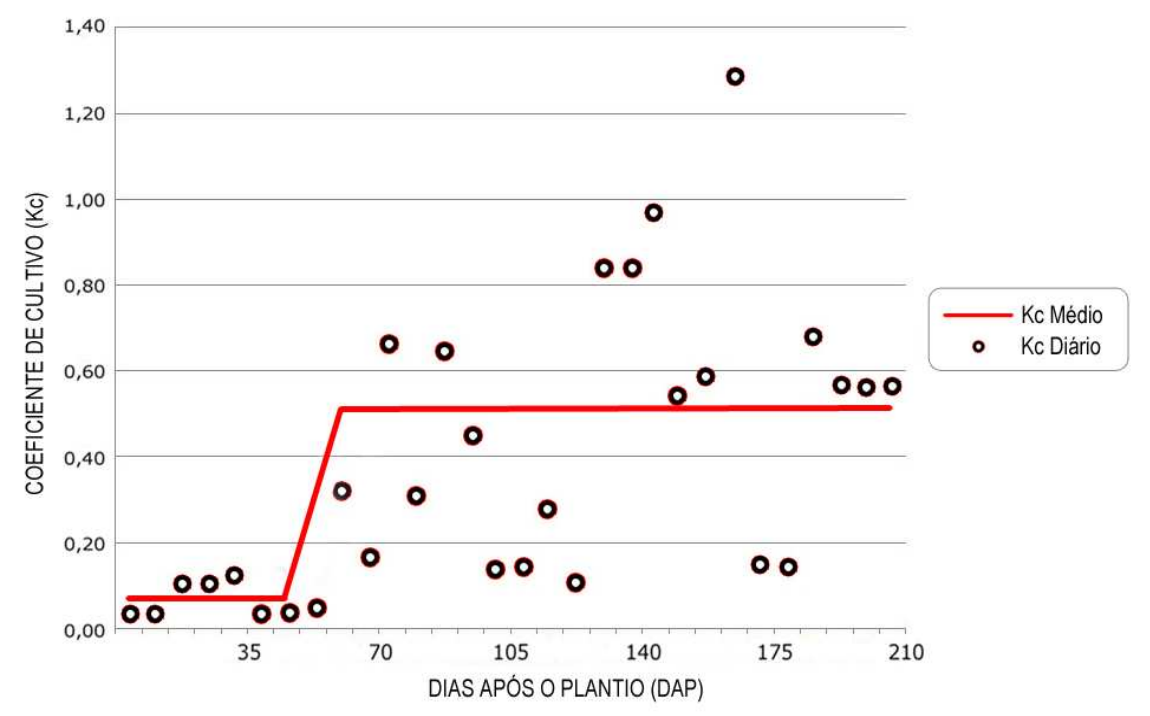

Figura 1. Curva do coeficiente de cultivo $(\mathrm{Kc})$ do abacaxi ornamental, observado em Pacajus, CE, 2014/2015.

Na Tabela 3 verificam-se os coeficientes de cultivo médios para as diferentes fases de desenvolvimento da cultura.

Tabela 3. Coeficientes de cultivo médios do abacaxi ornamental, nos diferentes estádios fenológicos, a partir da ETo estimada pelo método de Penman-Monteith em ambiente protegido. 


\begin{tabular}{ccc}
\hline Estádios Fenológicos & Períodos & Kc \\
\hline Inicial & 7 a 42 DAP & 0,07 \\
Intermediário & 43 a 63 DAP & 0,13 \\
Vegetativo & 64 a 210 DAP & 0,51 \\
\hline
\end{tabular}

${ }^{(1)}$ Dias após o plantio.

\section{CONCLUSÕES}

Os valores médios do coeficiente de cultivo (Kc) obtidos pela evapotranspiração de referências estimados pelo método de Penman-Monteith são de 0,07; 0,13; e 0,51 para o estádio Inicial, Intermediário e vegetativo respectivamente.

A evapotranspiração média da cultura foi de $4,29 \mathrm{~mm} \mathrm{dia}^{-1}$.

\section{REFERÊNCIAS}

AZEVEDO, P.V.; SOUZA, C. B. de; SILVA, B. B. da; SILVA, V. C. da. Water requirements of pineapple crop grown in a tropical environment, Brazil. Agricultural Water Management, v.88, n. 1 - 3, p. 201-208, 2007.

CARVALHO, A. C. P. P., de; SOUZA, F. V. D.; SOUZA, E. H. de. Produção de abacaxizeiro ornamental para Flor de Corte. Fortaleza: Embrapa Agroindústria Tropical, Série Documentos, 44 p. 2014.

MATOS, A. P.; CABRAL, J. R. S. Evaluation of Pineapple Genotypes for Resistance to Fusarium subglutinans. Acta Horticulturae, v. 702, p. 73-77, 2006.

SANTANA, J. N. de; SOUZA, O. P. de; CAMARGOS, A. E. V.; ANDRADE, J. P. R. Coeficientes de cultura do abacaxizeiro nas condições edafoclimáticas de Uberaba, MG. Revista Brasileira de Engenharia Agrícola e Ambiental, v.17, n.6, p.602-607, 2013. 